\title{
Surgical management of a complex case of Charcot arthropathy of the spine: a case report
}

\author{
Darshan Vora ${ }^{1} \cdot$ Cody D. Schlaff ${ }^{1} \cdot$ Michael K. Rosner $^{1}$
}

Received: 9 July 2019 / Revised: 1 August 2019 / Accepted: 5 August 2019

(c) International Spinal Cord Society 2019

\begin{abstract}
Introduction The authors present a case of a 55-year-old male with T10 complete paraplegia diagnosed with Charcot arthropathy of the spine (CAS).

Case presentation He presented to an outside institution with vomiting and productive cough with subsequent computed tomography (CT) and MRI imaging revealing L5 osteomyelitis and a paraspinal abscess. Given the patient's inability to remain in good posture in his wheelchair he underwent a multilevel vertebrectomy and thoracolumbar fusion. Due to multiple co-morbidities, surgical recovery was complex, ultimately requiring revision circumferential fixation.

Discussion CAS is an uncommon, long-term complication of traumatic spinal cord injury (SCI). Surgical management is often complex and associated with significant complications. Currently, a consensus on CAS prevention, specific surgical fixation techniques and post-surgical nursing care management is lacking. In this case report we provide our experience in the management of a complex case of CAS to aid in decision making for future neurosurgeons who encounter this sequela of traumatic SCI.
\end{abstract}

\section{Introduction}

Any insult to the vertebral column and spinal cord can lead to alterations of its function and vertebral column destruction. Pathologies such as Parkinson's Disease, diabetic neuropathy, syringomyelia, and neural tube defects have been shown to cause neuroarthropathy as a long-term sequela [1, 2]. Jean-Marie Charcot first described Charcot arthropathy in a patient in the 1800's. The first report of CAS was published in 1884 in a patient with tabes dorsalis, but with the advent of antibiotic treatments, CAS as a sequela of syphilis is rare [3, 4]. CAS is now a well-defined long-term complication following traumatic spinal cord injury (SCI), developing on average 17 years after injury.

The pathophysiology is not well understood, but believed to be a combination of neuro-trauma due to loss of sensation at the joint and neurovascular abnormalities due to

Darshan Vora

Darshanvora920@gmail.com

1 Department of Neurological Surgery, The George Washington University Hospital, The George Washington University School of Medicine and Health Sciences, Washington, DC, WA 20037, United States inflammation [5]. Recurrent infections increase joint destruction, and therefore more prevalent in wheelchairbound patients with poor nursing care [6]. Currently, no consensus exists on neurosurgical management of CAS. Here we describe the case of a patient with paraplegia who developed CAS following a traumatic SCI complicated by a complex hospital course.

\section{Case presentation}

\section{History and examination}

A 55-year-old male with T10 complete paraplegia was transferred to our institution on broad-spectrum antibiotics from another facility after presenting with 2 days of emesis and productive cough. He was being treated for a "bone infection" and lateral abdominal wall abscess with drain placement, which was mal-positioned on radiologic imaging. Eleven years ago, the patient was injured from a gunshot wound leaving him with paraplegia. He had neurogenic bowel and bladder dysfunction managed with an indwelling urethral catheter and sigmoid colostomy. Additionally, the patient had poor skilled nursing care, resulting in non-healing sacral and lower extremity ulcers. 
Physical exam on transfer was remarkable for inability to sit upright in his wheelchair, minimal expiratory wheezing, increased green/brown watery ostomy output, multiple ulcers, sacral wound with significant depth beyond the spinal column, tenderness to palpation on the thoracic spine, and exposed wound on the lumbar spine with an overlying hard palpable mass. Neurologic exam was notable for flaccid paralysis in the bilateral lower extremities and a T10 sensory level at the umbilicus. Medical history was notable for COPD, left lower extremity osteomyelitis, Clostridium difficle colitis, MRSA and IV drug use. Lab values were notable for elevated inflammatory markers (ESR/CRP), leukocytosis, and hypoalbuminemia.

\section{Initial imaging}

Outside CT abdominal/pelvis with contrast demonstrated a large sacral pressure ulcer with underlying fragmentation and destruction of the bilateral lumbosacral junctions; osteomyelitis of the left ischium and right iliac bone; and 6$\mathrm{cm}$ left lateral wall abscess with osteomyelitis at the left eleventh rib and dislodged drainage catheter outside of the fluid collection (Fig. 1).

Subsequent non-contrast CT demonstrated severe T11 vertebral body deformity, a ballistic tract extending into the spinal canal and articular process of $\mathrm{T} 12$ and retained ballistic fragment in the left T12 paraspinal soft tissue. A sacral pressure ulcer extended into the lumbosacral junction causing osteomyelitis of the right iliac crest, L2/L3 vertebral bodies, and sacrum (Fig. 1). Imaging was also notable for complete destruction of the L4 and L5 vertebral bodies, periosteal destruction, and sclerosis of the L 3 vertebral body extending into the L2-3 disc space (Fig. 1).

\section{Index surgical treatment}

On hospital day (HOD) 10, the patient underwent an index surgery of L4 and L5 vertebral column resection, posterior L3-S1 interbody fusion utilizing cage and bone graft, T2iliac posterior spinal fusion with instrumentation and bone graft, and L1-L2 and L2-L3 osteotomy. As patient was wheelchair bound, fusion to $\mathrm{T} 2$ was done to optimize upright positioning. A wound VAC was placed inferiorly over the sacral region and iliac crest bilaterally up to L3. The top three-quarter portion of the wound was closed in the usual fashion. Per patients request, the retained bullet in the paraspinal musculature was removed. Upper extremity somatosensory and motor evoked potentials performed throughout the case showed no changes from baseline. All thoracic screws showed greater than $8 \mathrm{mAs}$ and intraoperative $\mathrm{O}$-arm demonstrated appropriate positioning of implants and screws (Fig. 2).

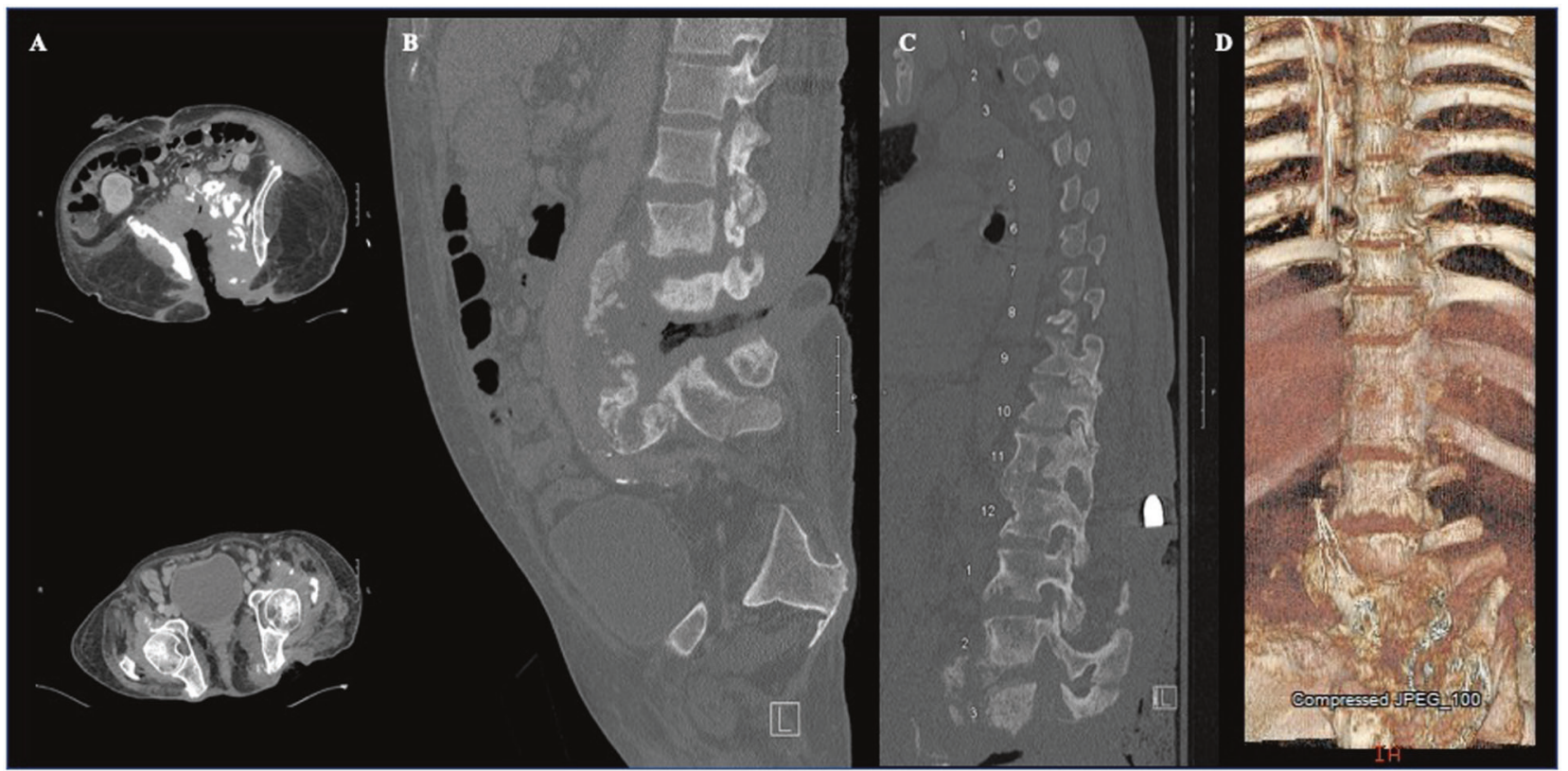

Fig. 1 Radiographic images from outside hospital. Imaging on presentation from OSH showed a Axial CT imaging demonstrating a large penetrating decubitus ulcer extending into the lumbosacral junction and along the right iliac crest with associated osteomyelitis involving the right iliac crest and sacrum. Additionally, there was evidence of a left ischial ulcer with associated left hip effusion. b Sagittal CT imaging demonstrating complete dissociation of the lumbosacral junction with heterotopic ossification. There is also evidence of complete destruction and collapse of the L4 and L5 vertebrae with partial destruction of L3. c Sagittal CT imaging revealed ballistic bony changes involving T9 -T11 vertebral bodies with a retained ballistic fragment at the T12 soft tissues. d Sagittal 3dimensional reconstruction of the thoracolumbar spine demonstrating a Charcot joint at the L1-L2 vertebral level with an associated dextroconvexity centered at the destroyed L4-L5 vertebrae 


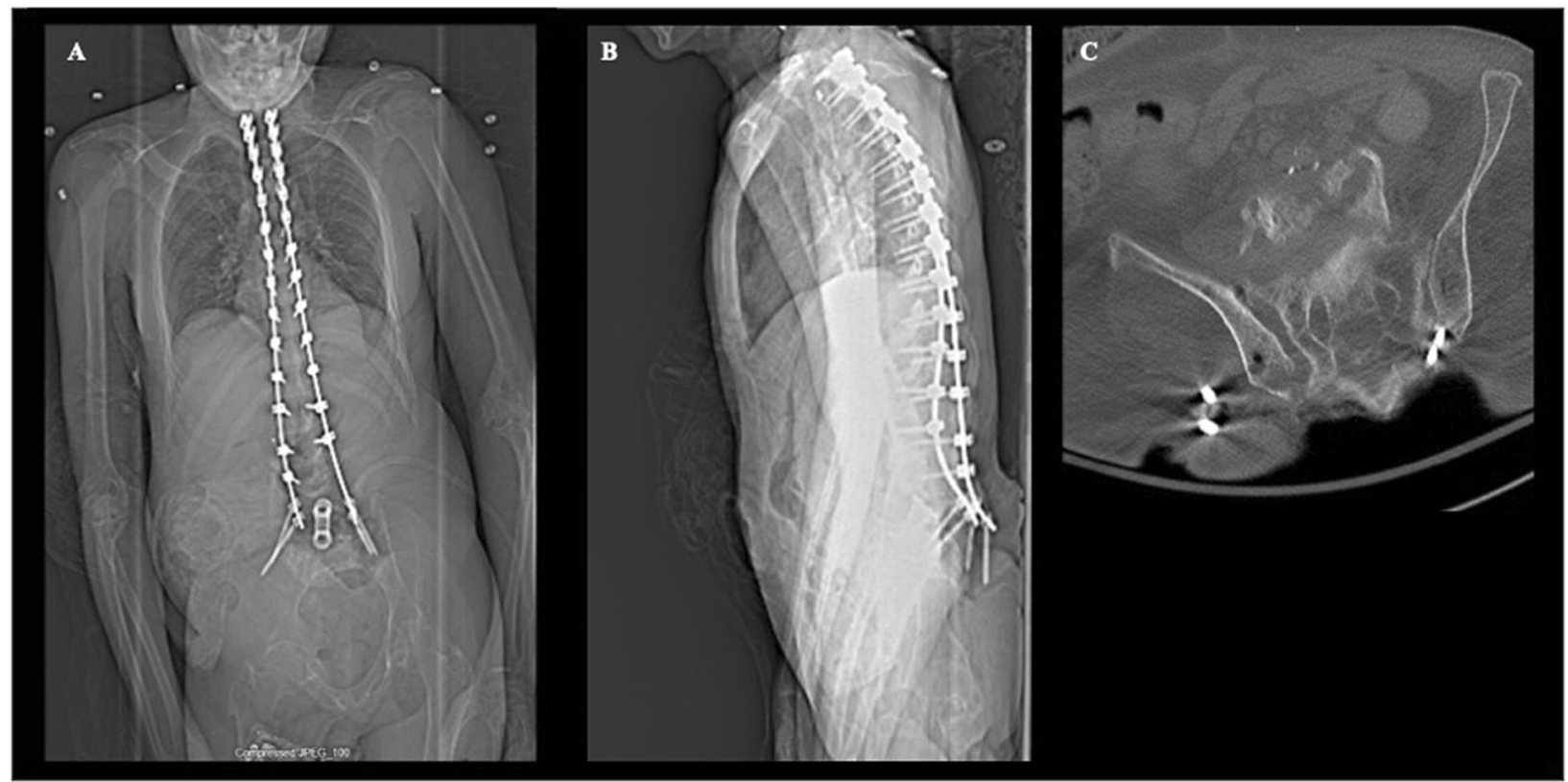

Fig. 2 Index surgery post-operative imaging. a Index surgery postoperative CT scout imaging showing posterior thoracolumbar fusion extending from the $\mathrm{T} 2$ vertebrae to iliac with associated corpectomy cage at the L4 and L5 levels. b Coronal CT scout imaging demonstrating the right-sided iliac screws situated in the subcutaneous soft tissue overlying the right gluteus maximus. c Axial CT imaging re-demonstrating the pulled-out right-sided iliac screws
Fig. 3 Post-operative revision surgery imaging. CT scout imaging of the revision surgery demonstrating replacement and upsizing of original iliac screws with an additional fusion construct lateral to the original fusion construct extending from the T10 vertebral level to the S2 vertebral level

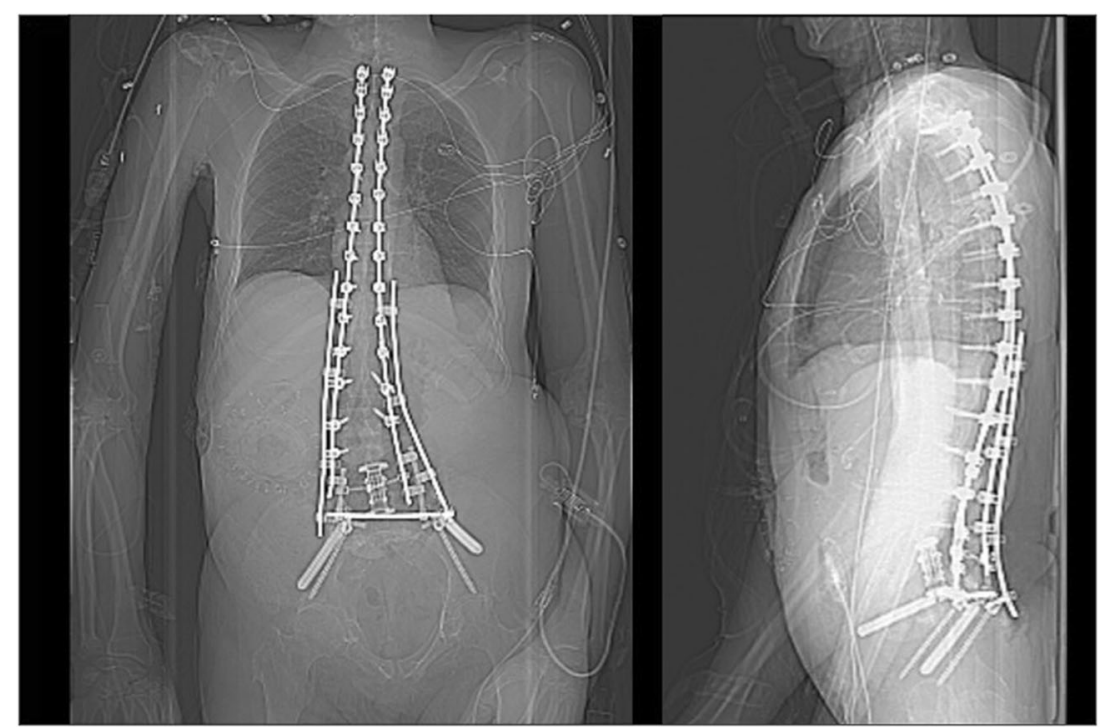

\section{Post-operative course}

Post-operatively, the patient remained intubated due to respiratory insufficiency secondary to prolonged anesthesia time and persistent hypotension requiring pressure support and transfusion. Intraoperative estimated blood loss was $700 \mathrm{cc}$ and the patient received 2.7L crystalloid, 6 units of packed red blood cells (PRBCs), and 2 units of fresh frozen plasma (FFP). The patient was extubated on post-operative day (POD) 1 but remained persistently hypotensive and tachycardic. Patient had a complicated and difficult post- operative course. Due to nephrotoxicity from vancomycin while hypotensive, the patient developed a non-oliguric acute kidney injury and hydronephrosis from a malpositioned indwelling catheter. During a routine wound VAC change on POD 20, the patient felt a "pop" and noticed his distal fixation present. Imaging revealed pullout of the iliac screw fixation and loss of distraction with the interbody cage (Fig. 3).

On HOD 30/POD21, the patient returned to the operating room for a revision T2-iliac posterior spinal fusion with instrumentation, L3-S1 anterior interbody fixation, 
implantation of new bilateral iliac screw, iliac crest cortoss fixation, and transiliac bar cross link fixation (Fig. 3). A wound VAC was placed over the entire surgical field.

Patient remained in the hospital for IV broad-spectrum antibiotics in consultation with infectious disease service and bi-weekly wound VAC changes. Despite his co-morbidities, the surgical site healed well. However, was deemed a non-optimal candidate for debridement and skin flap closure of his sacral pressure ulcer. The patient was discharged home 5 months from initial presentation with home health care and indefinite IV and oral antibiotics.

\section{Discussion}

Patients who develop CAS often present with back pain, wheelchair instability, or autonomic dysreflexia inferior to the level of injury [7, 8]. Signs of CAS development often do not manifest for decades after injury and may be overlooked [9].

Aebli et al. has investigated the clinical presentation and early detection of CAS in high risk patients and recommends radiological screening in patients with paraplegia to identify disc space narrowing [9, 10]. Surgical fusion and instrumentation has been reported to provide positive outcomes and allow patients to return to their previous level of activity; however, the risk of re-occurrence in patients with paraplegia warrants careful consideration prior to intervention [11-13] .

Surgical treatment is known to have a high rate of complications and there are no established recommendations for optimal surgical approach [14]. Recent case series recommend circumferential fixation for patients with multiple level involvement, whether performed as a single-stage or multistage operation $[5,15]$. Use of bone morphogenetic protein and four-rod lumbopelvic fixation showed favorable outcomes in 23 patients [16]. Zyck et al. reports relief of autonomic dysreflexia symptoms after four-rod thoracolumbar posterior fusion in a patient with C6 quadriplegia [17].

A case series of nine patients demonstrated successful fusion in all patients, eight of which underwent circumferential fusion, with an improvement in functional status in all patients [18]. However, successful posterior fusions have been observed in patients with only mild bone destruction [19]. Although one-staged anterior and posterior fusion has shown the most favorable outcomes, two-staged fusion in posterior instrumentation followed by anterior bone graft can be used in patients with extensive co-morbidities who cannot tolerate prolonged operative times [19]. Few studies have reported long-term follow-up.

Haus et al., demonstrated an average follow-up of 14.3 years and noted that $75 \%$ of patients who received a fusion developed another Charcot joint requiring revision surgery [20]. Thus, the authors recommended conservative treatment in those whose dysreflexia symptoms can be managed non-surgically [21]. In contrast, Grassner et al., advises against posterior-only fusions due to a high rate of pseudoarthrosis, as seen in our patient, and wound healing delays [22]. Unique to our patient, Suda et al., recommends percutaneous external spinal fixation and wound irrigation for unstable spine lesions with infection, followed by definitive surgical management when infection resolves [23].

Prevention is centered around decreasing the force applied to bony elements of the spine. Standaert et al., cautions against long fusions that end at the thoracolumbar junction, or procedures that increase upper trunk mobility as these increase stress on the lower spine [24]. Post-operatively, positioning and turning patients should be centered around decreasing the stress on the spine and the patient should be turned every 2-3 h. Initially, turning is restricted to 30 degrees on each side with the two-person logrolling technique, one supporting the shoulders and buttocks, while the other supports the knees and waist; this reduces twisting of the spine [25].

Proper patient turning is often neglected as the techniques are resource heavy, but minimizing twisting, lifting and bending can drastically improve outcomes [26, 27].

CAS is a long-term complication in patients with traumatic SCI. Surgical management is complex with a high level of complications. A multi-modal treatment approach is necessary as patients often have multiple co-morbidities limiting wound healing and successful arthrodesis. Prevention of CAS by appropriate nursing care and early mobilization is crucial. Surgeons report favorable outcomes following one-staged circumferential fusion in good surgical candidates. Our patient did not undergo initial circumferential fusion. In hindsight, he may have benefited from this approach due to extensive spinal disease and co-morbidities. Further research is necessary to decrease morbidity in CAS patients and establish a consensus for surgical management.

Acknowledgements We thank the faculty and staff of the Department of Neurological Surgery for their assistance in management of this patient and their support in drafting this manuscript.

\section{Compliance with ethical standards}

Conflict of interest The authors declare that they have no conflict of interest.

Publisher's note: Springer Nature remains neutral with regard to jurisdictional claims in published maps and institutional affiliations.

\section{References}

1. Loriaut $\mathrm{P}$, Rozenberg S, Boyer P, Dallaudière B, Khiami F, Sariali E, et al. Charcot spine and Parkinson's disease. Case Rep Orthop. 2014;2014:631346. 
2. van Eeckhoudt S, Minet M, Lecouvet F, Galant C, Banse X, Lambert M, et al. Charcot spinal arthropathy in a diabetic patient. Acta Clin Belg. 2014;69:296-8.

3. Charcot J. On some arthropathy which apparently depend on a brain or spinal cord lesion. Arch Physiol Norm Pathol. $1868 ; 1: 161-78$.

4. Kronig G. Spondylolisthesis at a tabetic spine. Zeit Klin Med. 1884;7:165-70.

5. Lee D, Dahdaleh NS. Charcot spinal arthropathy. J Craniovertebr Junction Spine. 2018;9:9-19.

6. Goodwin CR, Ahmed AK, Abu-Bonsrah N, De la Garza-Ramos R, Petteys RJ, Sciubba DM. Charcot spinal arthropathy after spinal cord injury. Spine J. 2016;16:e545-546.

7. Morita M, Iwasaki M, Okuda S, Oda T, Miyauchi A. Autonomic dysreflexia associated with Charcot spine following spinal cord injury: a case report and literature review. Eur Spine J. 2010;19 (Suppl 2):S179-182.

8. Selmi F, Frankel HL, Kumaraguru AP, Apostopoulos V. Charcot joint of the spine, a cause of autonomic dysreflexia in spinal cord injured patients. Spinal Cord. 2002;40:481-3.

9. Aebli N, Pötzel T, Krebs J. Characteristics and surgical management of neuropathic (Charcot) spinal arthropathy after spinal cord injury. Spine J. 2014;14:884-91.

10. Wirth CR, Jacobs RL, Rolander SD. Neuropathic spinal arthropathy. A review of the Charcot spine. Spine. 1980;5:558-67.

11. Brown CW, Jones B, Donaldson DH, Akmakjian J, Brugman JL. Neuropathic (Charcot) arthropathy of the spine after traumatic spinal paraplegia. Spine. 1992;17(6 Suppl):S103-108.

12. Luke DL, Bridwell KH. "Silent" spinal dislocation in a Charcot spine occurring postlaminectomy: case report and review of literature. J Spinal Disord. 1990;3:87-92.

13. Morita M, Miyauchi A, Okuda S, Oda T, Yamamoto T, Iwasaki M. Charcot spinal disease after spinal cord injury. J Neurosurg Spine. 2008;9:419-26.

14. Gibson JL, Vuong SM, Bohinski RJ. Management of autonomic dysreflexia associated with Charcot spinal arthropathy in a patient with complete spinal cord injury: Case report and review of the literature. Surg Neurol Int. 2018;9:113.
15. Devlin VJ, Ogilvie JW, Transfeldt EE, Boachie-Adjei O, Bradford DS. Surgical treatment of neuropathic spinal arthropathy. J Spinal Disord. 1991;4:319-28.

16. Jacobs WB, Bransford RJ, Bellabarba C, Chapman JR. Surgical management of Charcot spinal arthropathy: a single-center retrospective series highlighting the evolution of management. J Neurosurg Spine. 2012;17:422-31.

17. Zyck S, Toshkezi G, Pizzuti J, Marawar S. Four-rod instrumentation for treatment of Charcot spinal arthropathy causing autonomic dysreflexia: case report and literature review. Cureus. 2016;8:e850.

18. Vialle R, Mary P, Tassin J-L, Parker F, Guillaumat M. Charcot's disease of the spine: diagnosis and treatment. Spine. 2005;30: E315-322.

19. Suda Y, Shioda M, Kohno H, Machida M, Yamagishi M. Surgical treatment of Charcot spine. J Spinal Disord Tech. 2007;20:85-8.

20. Haus BM, Hsu AR, Yim ES, Meter JJ, Rinsky LA. Long-term follow-up of the surgical management of neuropathic arthropathy of the spine. Spine J. 2010;10:e6-16.

21. Moreau S, Lonjon G, Jameson R, Judet T, Garreau de Loubresse C. Do all Charcot spine require surgery? Orthop Trauma Surg Res. 2014;100:779-84.

22. Grassner L, Geuther M, Mach O, Bühren V, Vastmans J, Maier D. Charcot spinal arthropathy: an increasing long-term sequel after spinal cord injury with no straightforward management. Spinal Cord Ser Cases. 2015;1:15022.

23. Suda Y, Saito M, Shioda M, Kato H, Shibasaki K. Infected Charcot spine. Spinal Cord. 2005;43:256-9.

24. Standaert C, Cardenas DD, Anderson P. Charcot spine as a late complication of traumatic spinal cord injury. Arch Phys Med Rehabil. 1997;78:221-5.

25. Schoen DC. Adult orthopaedic nursing. Philadelphia: Lippincott; 2000.

26. Greenwood J, McGregor A, Jones F, Hurley M. Evaluating rehabilitation following lumbar fusion surgery (REFS): study protocol for a randomised controlled trial. Trials. 2015;16:251.

27. Lall MP, Restrepo E. The biopsychosocial model of low back pain and patient-centered outcomes following lumbar fusion. Orthop Nurs. 2017;36:213-21. 